\title{
Desarrollo de capacidades individuales y colectivas en estudios de posgrado y su incidencia en los territorios
}

Development of Individual and Collective Capacities
in Postgraduate Studies and their Impact on Territories

Desenvolvimento de capacidades individuais e coletivas em estudos de pós-graduação e sua incidência nos territórios

Cristina Girardo*

Sarah Martínez Pellegrini**

Luz Helena Salgado Locela**

Pablo Costamagna ${ }^{* * *}$

Recibido: 13 de marzo de 2015

Aprobado: 13 de julio de 2015

Doi: dx.doi.org/10.12804/territ34.2016.09

Para citar este artículo

Girardo, C., Martínez, P., Salgado, L. \& Costamagna, P. (2016). Desarrollo de capacidades individuales y colectivas en estudios de posgrado y su incidencia en los territorios. Territorios, 34, 215-236. Doi: dx.doi.org/10.12804/ territ34.2016.09

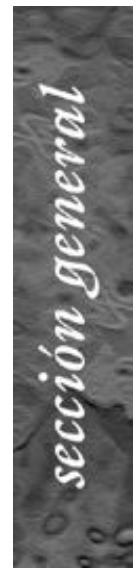

* Doctora en Pedagogía, Facultad de Ciencias de la Formación por la Universidad de Turin, Italia, Doctora en Estudios Latinoamericanos, Facultad de Ciencias Politicasy Sociales, UNAM, México. Adscrita a El Colegio Mexiquense. Correoelectrónico:c.girardo@ hotmail.com

* Doctora en Desarrollo e Integración Económica por la Universidad Autónoma de Madrid. Adscrita a El Colegio de la Frontera Norte. Correo electrónico: sarabm@colef.mx

*** Doctora en Ciencias Sociales con Especialidad en Estudios Regionales en El Colegio de la Frontera Norte. Adscrita a El Colegio Mexiquense. Correo electrónico:slocela@gmail.com **** Doctor en Estudios del Desarrollo de la Universidad del País Vasco. Adscrito $\Rightarrow$ 
Palabras clave

Territorio, educación, capacidades individuales, capacidades colectivas, estudios de posgrado.

Keywords

Territory, education, individual and collective capacities, postgraduate studies.

Palavras-chave

Território, educação, capacidades individuais, capacidades coletivas, estudos de pós-graduação.

\section{territarias 34}

\section{RESUMEN}

El presente estudio propone conocer la manera en la que los estudios del territorio brindan capacidades a los alumnos de educación superior, y la forma en la que los egresados contribuyen al desarrollo territorial desde su actual empleo, bajo el supuesto de que las capacidades individuales adquiridas en la formación educativa se transforman en colectivas y coadyuvan al desarrollo de los territorios. Se manejaron dos casos de estudio con información de los departamentos de docencia, y entrevistas semiestructuradas. Entre los resultados figuran que (a) los orígenes de las maestrías responden a demandas educativas locales, y tienen una fuerte vinculación con su entorno (desarrollo fronterizo y especialización municipal); (b) entre las capacidades de los egresados figuran un perfil interdisciplinario y habilidades cognitivas; (c) la constatación de la transformación de capacidades individuales a colectivas, si bien está condicionada a factores exógenos, los casos estudiados evidencian este pasaje sujeto siempre a los recursos en dónde se desarrollan.

\section{ABSTRACT}

A number of master degrees in Mexico include territory approaches in their programs in the main to provide specialized skills to their students so they can contribute on local development from their current jobs. The objective of this study lays in first place in determine the capacities that students from territory master degrees obtain, and in second place to show how their individual capacities transform into collective ones. Two case studies that make use of information from the departments of education, and semi-structured interviews were handled. The general results highlight: (a) territory master degrees respond to local education demands, and have a strong link to its with local context; (b) among the graduates, capacities stand the interdisciplinary and cognitive skills; (c) the transformation from individual to collective capabilities is conditioned by exogenous factors, however the case studies shows that this process always depends on the local resources in which they were developed.

\section{RESUMO}

O presente estudo propõe conhecer a maneira em que os estudos do território oferecem capacidades aos alunos de educação superior, e a forma em que os formados contribuem ao desenvolvimento territorial desde o seu atual emprego, sob o suposto que as capacidades individuais adquiridas na formação educativa se transformam em coletivas e coadjuvam ao desenvolvimento dos territórios. Utilizaram-se dois casos de estudo com informação dos departamentos de docência, e entrevistas semiestruturadas. Entre os resultados figuram (a) as origens dos mestrados respondem a demandas educativas locais, e têm uma fonte vinculação com o seu entorno (desenvolvimento fronteiriço e especialização municipal). (b) Entre as capacidades dos formados figuram: um perfil interdisciplinar e habilidades cognitivas. (c) A constatação da transformação de capacidades individuais à coletivas se bem está condicionada a fatores exógenos, os casos estudados evidenciam esta passagem sujeito sempre aos recursos onde se desenvolvem. 


\section{Introducción}

Este documento recoge el resultado de un análisis de dos estudios de caso de posgrados en Maestría de Desarrollo Regional (Caso A) y Maestría en Ciencias Sociales, con especialidad en Desarrollo Municipal (Caso B), en cuyos objetivos se plantea contribuir a formar recursos humanos con capacidades de agencia en el territorio. El objetivo del análisis fue profundizar en la repercusión de estas modalidades de formación y brindar las capacidades necesarias para que sus egresados contribuyan, por medio de su empleo actual, al desarrollo territorial. En ese sentido, se parte del supuesto de que las capacidades individuales adquiridas en los procesos de formación educativa se transforman en capacidades colectivas, lo que genera procesos que coadyuvan al desarrollo territorial en diferentes niveles.

Desde el punto de vista de los enfoques, la integración del tema del desarrollo regional-local-municipal-territorial, tiene ya una trayectoria en México, tanto en actividades de docencia, como de investigación, extensión y difusión, si bien poco sistematizada (Girardo, 2007; Girardo, 2009; Girardo \& Suárez, 2010) y con mayor énfasis en estudios estrictamente regionales, rurales y estudios municipales, pero alejada de las visones más sistémicas y dinámicas que implican la construcción desde la perspectiva del territorio como un espacio socialmente construido.

A la fecha, sobre todo a partir de las propuestas de la globalización, en los escenarios académicos mexicanos ya se ha hecho presente la necesidad de impulsar el desarrollo territorial, retroalimentado algunos procesos de descentralización del estado.

Para desarrollar el trabajo, se presentará la información en el siguiente orden: primero se describirán los referentes conceptuales y la metodología utilizada; posteriormente se analizan los casos de estudio, sus objetivos, mapas curriculares, así como el perfil y seguimiento de egresados ${ }^{1}$; por último, se esbozan algunas reflexiones finales.

\section{Referentes conceptuales}

Uno de los cambios más significativos de la sociedad contemporánea es la función que el conocimiento y los saberes, tienen en los procesos de desarrollo territorial para mejorar el bienestar humano. Esto hace repensar el papel de la universidad y los centros de educación como actores clave, no solo en la producción y difusión del conocimiento, sino como articuladores de los actores que modelan el desarrollo en las distintas escalas territoriales.

Contemporáneamente, este debate involucra una revisión de los contenidos y orientación de la propia noción de desarrollo. En estos últimos tres decenios se destaca una manera multidimensional y multidisciplinaria de acercarse a sus definiciones e intervenciones. Se acentúan los enfoques que contribuyen a modificar las cosmovisiones anteriores, cuya posición centralista y economicista era portadora a la Universidad Tecnológica Nacional, Facultad Regional Rafaela. Correo electrónico:coboin@wilnet. com.ar

${ }^{1}$ Por razones de confidencialidad, se omiten los nombres de las instituciones a las que pertenecen los programas revisados.

territarios 34 217 
2 Se entiende por actor a todo sujeto, individual o colectivo, cuya identidad le permite reconocerse como colectividad o comparte de ella, y con capacidad de acción estratégica; esto es, con capacidad de identificar/ definir sus intereses y traducirlos en objetivos, diseñar un curso de acción (estrategia) para alcanzarlos y relativa autonomía (recursos y capacidades) para implementar ese curso de acción (Acuña, 2013).

\section{territarios 34} 218 de una visión del desarrollo de arriba hacia abajo, con una explicación monocausal.

Producto de este debate, las teorías del desarrollo humano, sustentable, comunitario, local-territorial, endógeno, del buen vivir (Alburquerque \& Dini, 2008; Sanahuja, 2010; Sen, 2002; Vázquez, 2005) cobran relevancia, poniendo el énfasis en el papel de los actores y las lógicas de construcción y orientación del proceso de cambio.

Para este trabajo se toma como referencia una conceptualización amplia del desarrollo territorial, entendido como proceso que toma como unidad de actuación el territorio, con movilización y participación de los actores territoriales, públicos y privados. El territorio, por lo tanto, es visto como un agente de transformación local, con particularidades propias, que requiere adaptarse a los grandes procesos de cambio que implica el desarrollo (Albuquerque \& Dini, 2008).

La necesidad de que los actores asuman en un proceso reflexivo, al estilo del propuesto por Storper (1997), la necesidad de moverse en sinergias con la transformación territorial lleva a plantearse qué tipo de capacidades son las necesarias para lograrlo. Así, se recupera el protagonismo del concepto de capacidades (tanto institucionales, de los actores, o sea individuales, como las capacidades colectivas), que ha tenido un recorrido histórico relacionado con enfoques, lenguajes y estrategias diversas.

Si bien, en términos generales, se entienden las capacidades como atributos latentes que los actores despliegan para emprender acciones encaminadas al desa- rrollo, así mismo, se hace referencia a un subconjunto de recursos o activos que permiten a personas, empresas, organizaciones e instituciones, en general, aprovechar al máximo otros recursos en los territorios (Bebbington, 1999). También, por capacidad se entiende la habilidad de comportarse de una manera, de cumplir una función o tarea y/o alcanzar un objetivo, es decir, una habilidad de agencia. Es preciso señalar cómo la noción de capacidad va de la mano con la noción de recursos, considerados como los bienes materiales, simbólicos, organizacionales, políticos, humanos, etc., que, precisamente, son necesarios para generar capacidades. En este sentido, como advierte Acuña (2013):

[...] "puede considerarse a los recursos como
indicadores observables de la presencia de
una capacidad (más allá que esta se efectivice
o no, dado que un actor ${ }^{2}$ puede contar con
capacidad para hacer algo y no ejercerla)"
(p. 42 ).

En algunas situaciones el actor puede querer ejercerla y estar condicionado por factores exógenos. En cuanto a las capacidades de recursos humanos, consideradas en este trabajo como capacidades individuales, se adquieren mediante todo el proceso que tiene lugar para logar la titulación de posgrado dentro del marco formal de la universidad (capacitación formal). Se supone que esta capacitación formal mejora la posibilidad de ser agentes de cambio, que van a cuestionar las dinámicas existentes y proponer nuevas maneras o formas de 
hacer las cosas (Girardo, 2012), y se suman a las que los alumnos de las maestrías analizadas en el trabajo ya habían adquirido en sus procesos de formación anterior. Se retoman algunos conceptos trabajados por otros autores como Gibbons (1998) y García y Fanelli (2001) que afirman cómo, dentro de la modalidad formal, la mayoría de las capacitaciones, ligadas directamente a la temática del desarrollo territorial, son capacitaciones que pueden estar generando formación a analistas o reconfiguradores del conocimiento, consultores y diseñadores de políticas. Este proceso se suele acompañar de reflexión, debate e investigación.

Las capacidades colectivas, en cambio, trascienden las individuales, ya que son las que se vinculan con la acción colectiva, las que efectivamente expresan la particularidad que va adquiriendo, por ejemplo, en los casos focalizados, características específicas de entornos, lugares, territorios. El ejercicio de la libertad individual, al contar con un mayor desarrollo de capacidades, no solamente impacta la propia vida, o las instituciones, sino que permite obtener mejores resultados, en el sentido que permite que la acción individual intervenga en la mejora de los acuerdos sociales, es decir, de la acción colectiva (Pereira, 2006; Sen, 1993). Así mismo, para que esta acción colectiva adquiera relevancia, se necesita del valor instrumental de otras capacidades, como las adquiridas, por ejemplo, en procesos formativos (formales e informales) de los individuos, o de las capacidades institucionales, así como las capacidades políticas (Madoery, 2014).
Steward (2005) sostiene que lo propio de estas capacidades colectivas reside en que no son reducibles a capacidades individuales y que pautan la acción colectiva. La interacción entre miembros de una comunidad, o de un grupo (incluyendo normas y valores), es importante para determinar los resultados y trasciende la acción individual. Este conjunto de reglas permite evaluar cuándo alguien es efectivamente parte de una práctica o no y, por lo tanto, cuando también es parte de una capacidad colectiva.

Precisamente, el problema de investigación se plantea este discernimiento, hasta qué punto las capacidades individuales, recibidas en los procesos de formación en la educación formal, se reconfiguran en capacidades colectivas con impacto en la mejora de los territorios.

\section{Metodología}

Para llevar a cabo la primera parte del análisis, se revisaron trabajos anteriores, ya mencionados, así como la identificación y sistematización de nueva información disponible sobre el tema ${ }^{3}$. Vale la pena señalar que el análisis de los casos de estudio no busca comparar ni evaluar el desempeño académico de las instituciones, su elección se basó en la disponibilidad de información por parte de los departamentos de docencia, la proximidad laboral de los autores que permite conocer a fondo su evolución, y su experiencia en la temática. Así mismo, es importante destacar que el objetivo es acercarse a los resultados en el territorio de
${ }^{3}$ Se agradecen los aportes discutidos en los distintosseminarios y trabajos sobre el tema, Costamagna, Pérez, y Spinelli (2013a); Costamagna y Pérez (2013) y Costamagna, Pérez y Spinelli (2013b). territarios 34 219 
${ }^{4}$ Es pertinente señalar que ambos programas analizados cuentan con una cantidad reducida de inscritos $y$ egresados anualmente, por ende, la muestra que se analiza corresponde con esta información.

${ }^{5}$ A la fecha, la institución cuenta con ocho programas de posgrado en total, todos en el Programa Nacional de Posgrados de Calidad del Consejo Nacional de Ciencia y Tecnología (PNPC).

territarias 34 220 esos programas a partir de la discusión de capacidades que parecen generarse en los egresados y no un seguimiento de egresados. En este sentido, sería útil plantear posteriormente un seguimiento temporal de estos sujetos para corroborar y mejorar la discusión con una visión dinámica de seguimiento de las acciones acorde con el enfoque de desarrollo territorial.

Para la segunda parte del trabajo, se recuperaron, sistematizaron y analizaron, los datos e información que brindaron las áreas de docencia y coordinación de maestrías de las dos instituciones involucradas, así como los resultados de entrevistas a algunos egresados para profundizar en el tema de sus procesos de aprendizaje. Del mismo modo, se realizaron diez entrevistas con el objetivo de recoger información sobre las diferentes experiencias de capacitación y se profundizó en dos historias de vida. Los entrevistados son coordinadores o docentes en estas carreras y alumnos egresados de dichas maestrías ${ }^{4}$. Las entrevistas semiestructuradas se realizaron a partir de un guión, lo que permitió recoger la información manteniendo, al mismo tiempo, una conversación abierta entre el entrevistador y el entrevistado (Kvale, 1983; Perry, 1998; Singh, 2008).

Se trata de una investigación exploratoria, en fase inicial, que trató de dar respuesta a los siguientes interrogantes: ¿̨uáles son las características de estas maestrías, origen, objetivos, contenidos, enfoques pedagógicos?, ¿qué criterios han imperado para el diseño de los programas y el perfil de alumnos que ingresan a las carreras sobre desarrollo territorial-local? Por otra parte, y en cuanto al seguimiento específico de egresados, interesó hacer la pregunta de ¿qué tipo de capacidades adquirieron en la maestría, que hoy pueden aplicar en sus trabajos?, ¿Cuál es la pertinencia de sus estudios en sus actuales trabajos?, ¿En qué medida ha favorecido la maestría para su inserción en el mundo laboral? ¿En qué medida las capacidades individuales adquiridas por los alumnos en los programas de posgrados estudiados se transforman en capacidades colectivas e inciden en el desarrollo de los territorios?

\section{3. ¿¿Dos programas, un objetivo, dos resultados?}

Para iniciar el análisis, es preciso hacer referencia al origen de ambos programas, ya que explica en gran medida por qué y qué orientación hacia el territorio refleja cada una de las formaciones que se brinda.

\section{Caso A}

La Maestría en Desarrollo Regional (MDR), caso A, comienza en 1984 como la primera maestría ofrecida por la institución en la que se imparte ${ }^{5}$. El propósito de la maestría va de la mano con los objetivos de la institución en su etapa inicial: hacer investigación sobre la frontera norte de México en ese entonces definida de manera muy funcional como el área física de territorio colindante con Estados Unidos. La necesidad era formar especialistas que tuvieran una visión regional para delimitar sus estudios y 
generar información en las zonas de interés. Después de un estudio de factibilidad en 1980, que consideró las condiciones económicas, demográficas y ofertas educativas en ambos lados de la frontera, se identificó la ciudad idónea para establecer un centro de estudios con actividad docente. La investigación generaría información valiosa y necesaria, estadísticas sociales y económicas que sirvieran para la toma de decisiones y posicionaría la importancia de tener centros de investigación fuera del centro del país para recuperar la información in situ. Se considera a la región, pero la perspectiva, como se aprecia, sigue siendo muy centralista de provisión de información.

Desde un principio, el interés de la maestría fue formar especialistas con un sentido práctico o instrumental, e insertarlos en el área educativa, en el sector público y en el productivo del norte de México. El perfil de ingreso era distinto al actual ${ }^{6}$. Después de quince generaciones es considerado el posgrado más consolidado de la institución ${ }^{7}$. Esta maestría fue, además, el origen de un Doctorado en Ciencias Sociales con Especialidad en Estudios Regionales que desde hace veinte años dicta también la institución.

A lo largo del tiempo, la maestría ha transformado su enfoque regional fronterizo a la par de la incursión en nuevos acercamientos a los problemas sociales y económicos, tema que se analiza con más detalle en la sección de análisis curricular. Las actividades docentes se han ido relacionando cada vez más con las de investigación y vinculación, que son las otras dos áreas sustantivas de la institución. Esto se ha considerado una forma de maximizar el impacto de los trabajos de los estudiantes en el territorio y de vincularlos a las redes locales desde el mismo proceso de investigación.

\section{Caso B}

La Maestría en Ciencias Sociales con especialidad en Desarrollo Municipal (MCS), caso $B$, se puso en marcha en el año de 1987 como maestría en Desarrollo Municipal. En 1990 (mediante un proceso de reconceptualización de sus objetivos, mapa curricular y perfil de egresados) la maestría se denominó en Ciencias Sociales con especialidad en Desarrollo Municipal; en enero de 1993 ingresó al Padrón de Programa de Excelencia para Ciencia y Tecnología. La Maestría es parte de un centro académico de excelencia que funciona desde 1986, donde se imparten posgrados ${ }^{8}$, y se hace investigación científica y difusión del conocimiento, por medio de publicaciones y actos académicos. La investigación ocupa un lugar preponderante en la institución, sus investigadores conforman la planta académica, otorgándole calidad y cientificidad a los estudios de posgrados que se dictan. Desde su origen, esta institución enfocó su interés hacia estudios locales, de desarrollo municipal, otorgándole una especialidad en el tema, tanto por la docencia como por investigaciones especializadas en estos temas (urbanos, metropolitanos, estatales, municipales), sin dejar de considerar sus vínculos con las escalas nacional e internacional.
${ }^{6}$ La mayor parte contaba con experiencia laboral previa al posgrado, su procedencia era de estados fronterizos $y$, a pesar de que en ese tiempo las terminaciones de estudio en el país eran muy desiguales, se les exigía baber hecho trabajo de tesis y tener su licenciatura concluida y existía una tendencia a que fueran egresados de estudios regionales (que en ese entonces se formaban en el Colegio de México) y de programas en ciencias sociales (economía, sociología, antropología, o ciencia política, por ejemplo).

Curricularmente, su evolución se ba ido formalizado con la obtención de titulos como Programa de Posgrado de Excelencia desde que CONACyT emite el Padrón Nacional de Posgrados en 1991 (que después cambia de nombre a Registro del Programa para el Fortalecimiento del Posgrado Nacional); posteriormente, se clasifica en la modalidad de Posgrado Consolidado, y desde 2013 tiene la clasificación de calidad internacional otorgada por CONACyT.

${ }^{8}$ Se imparten, además, en el Caso B otros tres posgrados de calidad: Doctorado en Ciencias Sociales, la Maes- $\Rightarrow$

territarias 34 
tría en Ciencias Sociales con Especialidad en Desarrollo Municipal y una Maestría en Historia, todos reconocidos en el PNPC.

\section{tersitarias 34}

La MCS, en función tanto de los cambios en los estudios sobre desarrollo localregional-territorial, como de las demanda del mercado, en 2009 dio inicio a un debate entre los investigadores ante la necesidad de actualizar sus planes de estudios, ampliando los seminarios de tesis y la oferta de materias optativas que dieran a los alumnos la posibilidad de realizar sus tesis de acuerdo con sus intereses. Así mismo, se ampliaron los objetivos formativos, se redefinieron los perfiles de ingreso y egreso, se reformularon los seminarios de tesis, se reorganizó el mapa curricular y el tiempo de dedicación del estudiante para su graduación oportuna.

Es loable señalar que esta especialidad en Desarrollo Municipal le otorga a esta institución un lugar pionero y casi único en las ofertas que se encuentran en México vinculadas al tema.

\section{Objetivos de los Programas}

Las figuras 1, 2, 3 y 4 resumen los objetivos de ambos programas de maestría. En ambos casos, estos objetivos reflejan el interés por formar especialistas en el análisis de fenómenos sociales con un enfoque territorial; sin embargo, en unos de los casos se prioriza con más énfasis la formación académica dirigida a la investigación, mientras que en el otro, si bien la investigación también es prioritaria, se combina con una visión más polivalente del egresado hacia la intervención. Esta situación puede estar muy relacionada con los contextos de las dos instituciones y los orígenes de ambos programas.

De manera consistente con lo anterior, los objetivos específicos de los programas de cada institución reflejan de nuevo esos perfiles generales, uno más centrado en el proceso académico y sus facetas, el otro algo más abocado a aspectos de intervención en el entorno.

De los objetivos propuestos por los dos programas de maestría, se deduce que ambas buscan la formación de recursos humanos por medio del fortalecimiento de competencias y capacidades individuales, poniendo énfasis en elevar la calidad profesional del individuo. Este objetivo es compartido con la búsqueda de incidir en los dos casos estudiados en la mejora de las condiciones de su entorno, así como en fenómenos sociales, políticos, económicos, territoriales y de gestión que repercutan en el desarrollo municipal y regional. Si se hace referencia a la producción de capacidades, se estaría ante la presencia de generación no solo de capacidades individuales sino además de la propuesta en sus objetivos de desarrollar capacidades y acciones colectivas para el desarrollo territorial.

En este sentido, la capacitación recibida en las maestrías tiene el potencial de convertirse en "un escenario para desarrollar un proceso de cogeneración de conocimiento" (Costamagna, Pérez \& Spinelli, 2013a; Costamagna y Pérez, 2013; Greenwood \& Levin, 2007). En los objetivos se identifican los actores del territorio que facilitan la inserción laboral de los egresados. 
Figura 1. Objetivo general Maestría en Desarrollo Regional, Caso A

$\begin{gathered}\text { Objetivo } \\ \text { General } \\ \text { Caso A } \\ 2014\end{gathered}$
Formar recursos humanos de alto
nivel académico con una visión inter y
multidiciplinaria en los campos de conocimiento
de las ciencias sociales con un enfoque regional.

Fuente: elaboración propia con base en información del área de docencia MDR.

Figura 2. Objetivos generales Maestría en Ciencias Sociales con especialidad en Desarrollo Municipal. Caso B

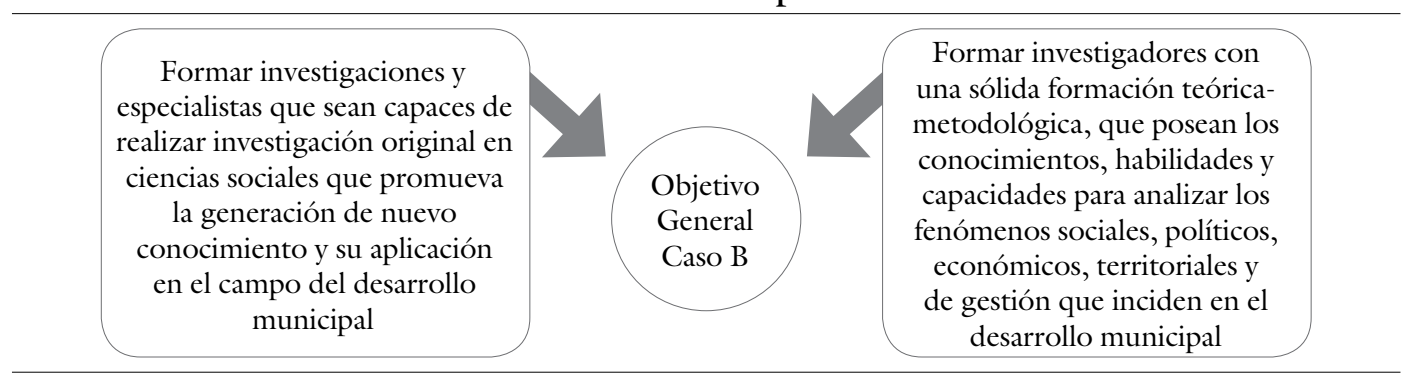

Fuente: elaboración propia con base en información del área de docencia MCS

Figura 3. Objetivos específicos Maestría en Desarrollo Regional, Caso A

$\begin{gathered}\text { Aplicar enfoques multidisciplinarios que permitan la } \\ \text { integración de conceptos y escalas espacio- temporales, asociados a } \\ \text { problemáticas regionales }\end{gathered}$
$\begin{gathered}\text { Generar } \\ \text { conocimiento } \\ \text { científico para abordar } \\ \text { los problemas actuales } \\ \text { del desarrollo regional }\end{gathered}$

Fuente: elaboración propia con base en información del área de docencia MDR. 


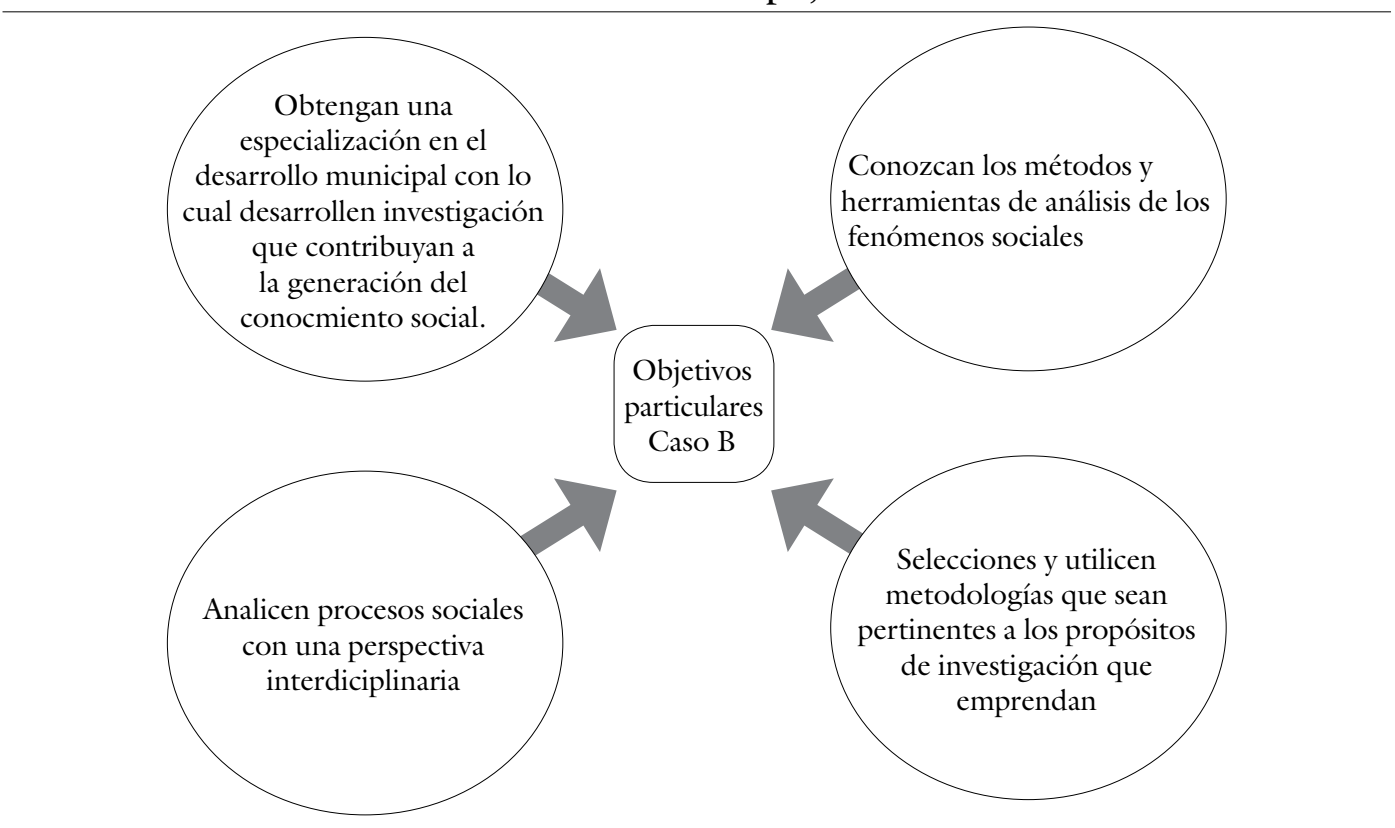

Fuente: elaboración propia con base en información del área de docencia MCs.

\section{Mapas Curriculares}

En el siguiente apartado se revisa si los programas contribuyen a la generación de conocimiento y capacidades individuales con el potencial de incorporarse posteriormente la acción colectiva en términos de políticas territoriales de desarrollo.

El Caso A siempre ha dividido su carga curricular en un área básica y otra de especialidad, la primera compuesta por cuatro ejes de estudio: (a) territorio y sociedad, (b) economía y territorio, (c) planeación regional y $(\mathrm{d})$ diseño y métodos de investigación territorial. El área de especialidad que (a) desarrollo urbano-regional, (b) sistemas productivos, (c) políticas y gestión territorial, posteriormente agrega un cuarto tema de especialidad: (d) migración e impactos en el territorio (figura 5).

Los cambios, tanto de nombres como de contenido en la estructura de las materias, obedecen a una reestructuración que comienza en 2005 con las adecuaciones propuestas por evaluaciones internas $y$ externas. Como producto de las observaciones se enfatizan aspectos conceptuales relativos al territorio y al desarrollo regional en las áreas básicas, el plan de estudios se modifica para que refleje un mayor énfasis en aspectos territoriales, se reduce la carga 


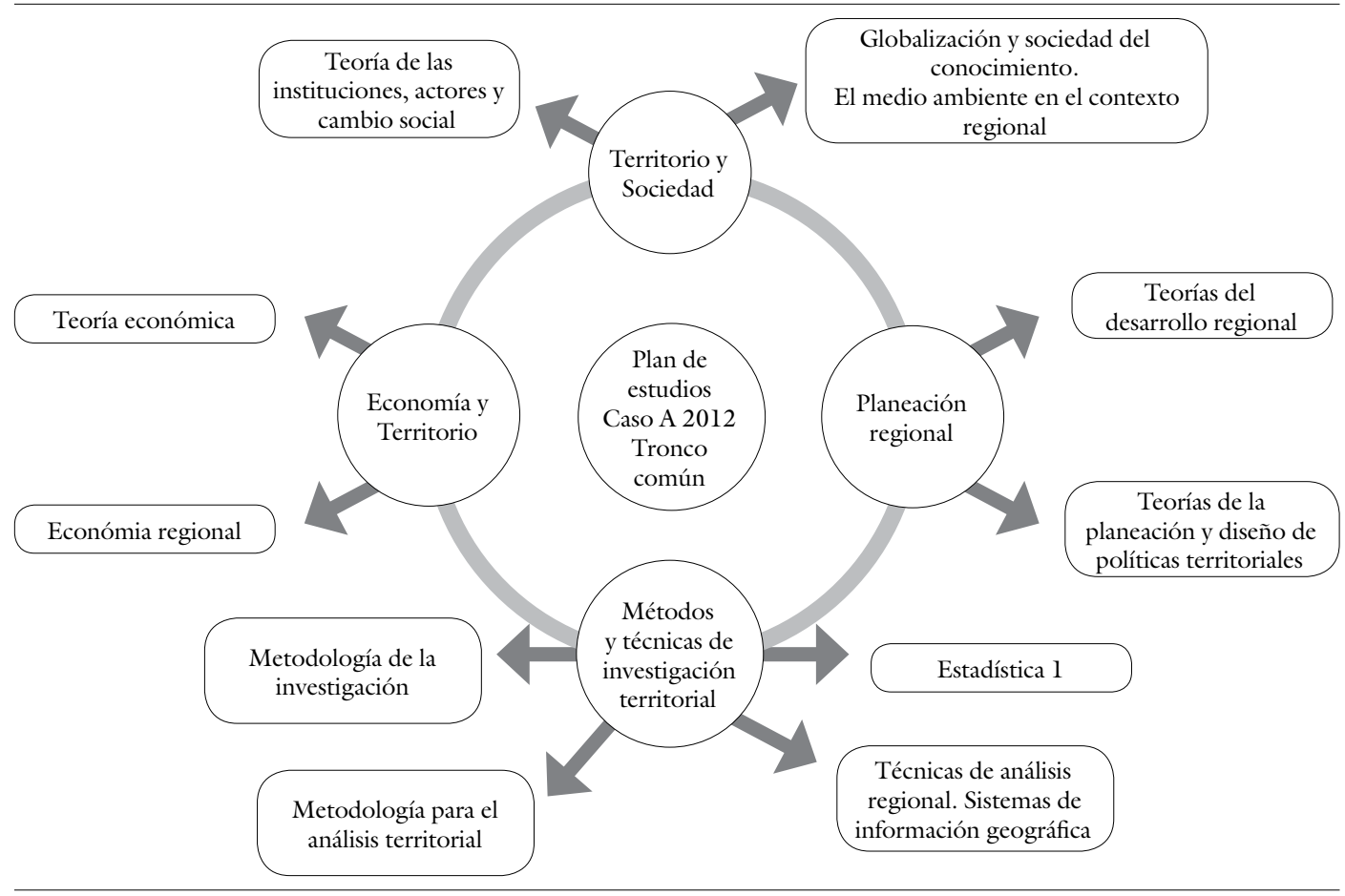

Fuente: elaboración propia con base en información del área de docencia MDR.

académica de los estudiantes, las especialidades temáticas se refuerzan y se cambia de un esquema trimestral a semestral que les permite a los estudiantes aprovechar oportunidades de intercambio, trabajo de campo y tener mayor tiempo para la redacción de la tesis. No menos importante, se rescata el estudio de teorías de cambio social, instituciones y actores, y se incorpora la dimensión ambiental, así como los temas de globalización y sociedad del conocimiento, discusiones transversales que anteriormente no presentaban un peso en el diseño curricular básico.
La retroalimentación interna del programa ha sido parte esencial para su continua transformación. La experiencia y el trabajo de los coordinadores plasmado en documentos oficiales, el debate entre organismos ya establecidos (comisión académica y grupos de las especialidades temáticas), incluso la discusión de crear una Academia sobre Territorio para discutir las fronteras del conocimiento en el análisis de los territorios, y los traslapes entre dimensiones expresados en debates sobre gobernanza, globalización, polarización económica, movilidad social, etc. (Almaráz, 2010). 
Toda esta autocrítica interna ha contribuido a la evolución de los planes de estudio cuya concepción de región fronteriza se transforma desde una frontera definida en términos de un espacio geográfico opuesto al centro del país definido a partir de una perspectiva funcional, al de una construcción social surgida a partir de las características del propio territorio en una visión dialéctica global-local y endógeno-exógeno (Martínez, 2014).

En el caso B, la Maestría ha llevado a cabo la actualización del plan de estudios, y como se ha observado en la descripción de los objetivos, el fin último de esta es formar investigadores capaces de desempeñarse en instituciones académicas, organizaciones no gubernamentales y de la sociedad civil, así como en organismos del sector público en todos los niveles de gobierno. En este sentido, la preocupación que se trae en este trabajo sobre la incidencia en los territorios de sus egresados o la pertinencia de la formación para llevar a cabo sus trabajos, obliga a revisar si contribuyen efectivamente a la generación de conocimiento o a la consecución de programas y políticas encaminadas al logro de procesos de desarrollo municipal (figura 6).

Figura 6. Mapa curricular Maestría en Ciencias Sociales con especialidad en Desarrollo, Caso B

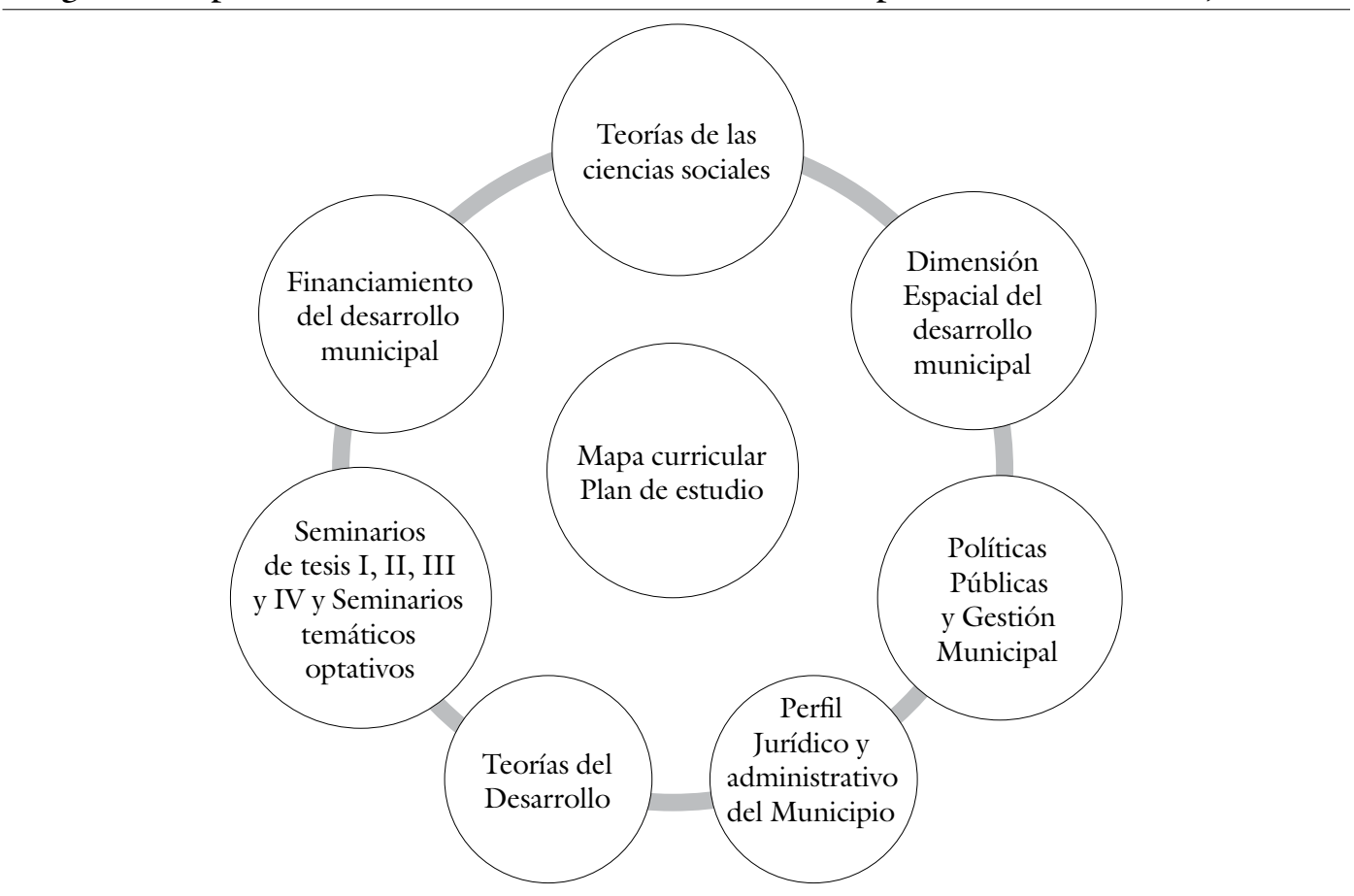

Fuente: elaboración propia con base en información del área de docencia MCS. 
Dentro de las capacidades que la maestría del caso B, se propone resaltar, mediante la capacitación, las capacidades analíticas, conceptuales, metodológicas e instrumentales para la investigación, la docencia y la intervención, con preferencia en materia de desarrollo municipal, pero siempre considerando las demás escalas (estatales, nacionales e internacionales). Sin embargo, en el plan de estudio, este aspecto está condicionado por las asignaturas dirigidas específicamente al ámbito municipal. Esto contrasta con la maestría del caso A en la que se plantea una lógica de construcción de los territorios, en la que, sin embargo, existen algunas debilidades en la incorporación de nociones geográficas en esta discusión.

Sin embargo, y a pesar de que en este tipo de maestrías vinculadas a la educación formal, la mayoría busca sobre todo el fortalecimiento de las competencias y capacidades individuales, cuyo objetivo es elevar la calidad de la actividad profesional del individuo (Basabe \& Cols, 2007), en ambas maestrías, se tiene en cuenta en su plan de estudio, en sus objetivos y en el tipo de capacidades que se brindan la incidencia en capacidades colectivas, es decir, lograr que los conocimientos adquiridos se transformen en acciones concretas en los territorios en donde actúan.

La mayoría de las capacitaciones incluyen contenidos de metodología para la investigación, y se ha proporcionado una ampliación a las horas dedicadas a seminarios de tesis y, sobre todo, a la oportunidad de seleccionar materias optativas, que tengan que ver con los intereses concretos de los alumnos. Es indudable que quienes movilizan los temas son los docentes e investigadores en los cuales se apoyan las maestrías, esto facilita que un tema se posicione en el programa, por ende, es importante destacar la importancia de entender la perspectiva y aproximación que existe detrás de las capacitaciones y su influencia en esta (Carreño, 2011 ; Costamagna, 2011; Díaz, 2008).

\section{Perfil y seguimiento de egresados}

Los dos programas cuentan tanto con la definición del perfil de egresados que pretenden alcanzar al finalizar sus estudios, con un programa institucional gestionado por la coordinación de docencia, basado en un proceso continuo y permanente que les permite establecer un contacto con sus egresados a fin de recaudar información que conduce a la verificación de la pertinencia del currículo, también al ofrecimiento de capacitación y actualización en relación con los objetivos de cada maestría, así como su inserción ante el sector laboral productivo, que les permita desempeñarse en la sociedad y constatar los niveles de satisfacción alcanzados por su formación profesional, que a su vez permite corregir hasta donde sea necesario y conveniente los planes de dichos programas.

En este apartado se reporta en base con el perfil supuesto y en los datos duros de seguimiento de egresados los resultados obtenidos en cada Programa estudiado. 
${ }^{9}$ El egresado [...] "contará con una formación integral que le permita abordar y entender la complejidad de los problemas del desarrollo regional con un enfoque interdisciplinario, de manera que sea capaz de identificar los componentes básicosy ofrecer soluciones pertinentes a los mismos. Con esta formación podrá desenvolverse con éxito tanto en dependencias públicas como privadas, en organismos no gubernamentales y en instituciones de investigación $y$ docencia involucradas con el diagnóstico, la planeación y la conducción del desarrollo regional" ( $m d r$, 2002-2010).

territarias 34 228

\section{Caso A}

En la revisión documental se observó que en el periodo 2002-2010 el Perfil del Egresado de la MDR no muestra cambios ${ }^{9}$. La primera parte del perfil muestra los resultados de la maestría en términos cognitivos, sus características y finalidad. La formación integral se entiende como instrumento del pensamiento necesario para abordar $y$ entender la complejidad de los problemas del desarrollo regional, su principal característica es un enfoque interdisciplinario y su finalidad es identificar los componentes básicos y ofrecer soluciones a los problemas del desarrollo regional. La segunda parte, es la forma y el ámbito de trabajo del egresado de la maestría, este "podrá desenvolverse con éxito tanto en dependencias públicas como privadas, en organismos no gubernamentales y en instituciones de investigación y docencia", y también se señalan las habilidades más destacadas en la práctica de su profesión "diagnóstico, planeación y conducción del desarrollo regional".

A partir de 2012, el perfil del egresado sufre modificaciones, eliminan la connotación de éxito y el ámbito de trabajo del egresado, y se plantean dos líneas generales: conocimientos y capacidades (tabla 1). La línea de conocimiento detalla de manera más puntual las herramientas cognitivas mencionadas en el perfil anterior (enfoques teóricos conceptuales y metodológicos, fenómenos territoriales y regionales), agrega un pensamiento crítico y reflexivo, así como el estado del arte de los subsistemas. La segunda línea de capacidades menciona habilidades de corte más general como cooperación, trabajo en equipo y coordinación de grupos de investigación, así como habilidades que requieren de un entrenamiento temático especializado $\mathrm{co}^{-}$ mo formulación y diseño de estrategias en los ámbitos local y regional, conducción de proyectos de investigación en estudios regionales y manejo de esquemas de evaluación de políticas territoriales.

El análisis del perfil del egresado para cada uno de los casos se hizo con una entrevista a profundidad en la que se recupera su opinión y experiencia en los siguientes tres puntos: (a) Formación integral-consolidada, (b) Enfoque interdisciplinario y (c) Articulación de los egresados a las necesidades territoriales.

Formación integral: Este concepto no está definido en el perfil de egresado de la MDR (2002-2010) y, para el egresado, se refiere a "una propuesta de acción, es decir, incluye tareas que deben evidenciarse, a saber: formar tanto intelecto como cuerpo, sensibilidad e identidad ética y ciudadana". La parte de formación del intelecto se cumplió, existe una gran cantidad de lecturas que los alumnos deben asimilar y que son objeto de evaluación, lo que se demuestra en el número de alumnos dados de baja. También considera que el intelecto implica capacidades como análisis, argumentación y deliberación, que se evidencian en la elaboración y defensa de una tesis. La sensibilidad e identidad ética son intangibles y se observan en las relaciones de amistad y compromiso que surgen entre estudiantes, con profesores y directores de tesis. De 
acuerdo con su definición de formación integral, el egresado la MDR concluye que es un posgrado que procura la formación integral de sus alumnos.

Enfoque interdisciplinario: el egresado comenta que para el diálogo con otras disciplinas que se interceptan en el estudio de los fenómenos regionales este debe tener "habilidades para el debate y atención a las diferencias conceptuales y metodológicas" (Comunicación personal Caso A, 2014) y menciona la importancia de mantener una clara apertura a la articulación de los objetos estudiados. La MDR, comenta el egresado, desarrolla habilidades de debate académico en ejercicios orales donde el alumno hace una defensa del avance de tesis ante una mesa de especialistas. Así mismo, las exposiciones orales y trabajos en equipo obligan a los alumnos (provenientes de diversas profesiones) a manejar críticas, defender sus puntos de vista y concertar esfuerzos como equipo para obtener productos de calidad. Actualmente, el egresado es docente de una universidad federal en donde se relaciona con profesionales de otras áreas, y la formación interdisciplinaria es una herramienta que le ha permitido una integración profesional e interactuar con otros docentes.

Articulación de los egresados a las necesidades territoriales: El egresado manifiesta que, a pesar de que la formación de la MDR le permitió tener elementos para insertarse en un ejercicio académico profesional de forma relativamente sencilla, los aspectos externos son los que dificultan su ámbito de acción profesional. La pertenen- cia a un sindicato le significa, por un lado, una seguridad en sus derechos laborales, pero, por otro, no está ajeno a prácticas corporativas acompañadas con autoritarismo y represión. También se enfrenta a prácticas centralistas, ya que en una universidad federal donde trabaja las estrategias o programas educativos son determinados desde la rectoría, lo que para el egresado tiene ventajas y desventajas ${ }^{10}$.

Para el caso de la Maestría Caso A, la figura 7 ilustra que la incorporación de los egresados es en su mayoría en la academia (64\%), y en menor medida en el sector gobierno u organizaciones públicas (36\%). Con mayor detalle, los egresados se encuentran en cargos como investigadores y/o docentes en universidades públicas.

Figura 7. Tipo de empleo de egresados 2013, Caso A

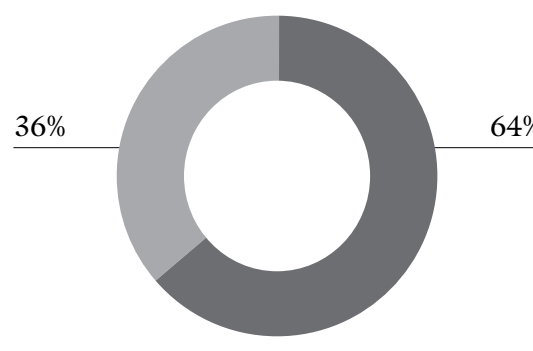

- Académico

- No académico

Fuente: elaboración propia con base en información del área de docencia MDR.

\section{Caso B}

El perfil del egresado de la MCS en el periodo 2002-2010, publicado en la página
10 "Ventajas por la envergadura y la visión de las politicas y los programas, sin embargo, también implica una traba en términos de la flexibilidad y prontitud de las respuestas a las demandas locales o regionales por parte de las unidades [...] a lo largo del territorio nacional" (Comunicación personal Caso A, 2014).

territarios 34

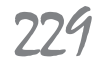




\begin{tabular}{|c|c|}
\hline MDR 2012- 2014 & MDR 2012- 2010 \\
\hline \multicolumn{2}{|c|}{ Conocimiento de: } \\
\hline $\begin{array}{l}\text { Enfoques teórico-conceptuales y metodológicos de corte } \\
\text { multidisciplinario, para analizar y explicar problemáticas } \\
\text { regionales y sus relaciones relevantes con los procesos } \\
\text { económicos, políticos, sociales, ambientales y culturales } \\
\text { que las producen. }\end{array}$ & \multirow{3}{*}{$\begin{array}{l}\text { Visión consolidada sobre la importancia } \\
\text { de las ciencias sociales en el estudio de } \\
\text { la transfomación social y en particular } \\
\text { la interacción de los actores relevantes } \\
\text { en un espacio municipal. }\end{array}$} \\
\hline $\begin{array}{l}\text { Los fenómenos territoriales y regionales acordes a un } \\
\text { pensamiento crítico y reflexivo para su análisis, sintesis } \\
\text { e interpretación. }\end{array}$ & \\
\hline $\begin{array}{l}\text { Del estado del arte de los campos teórico-metológicos en las } \\
\text { áreas de especialización temática: Desarrollo urbano-regional. } \\
\text { Sistemas productivos, politícos y gestión territorial, y } \\
\text { migración e impactos en el territorio. }\end{array}$ & \\
\hline \multicolumn{2}{|l|}{ Capacidades de: } \\
\hline $\begin{array}{l}\text { Formular y diseñar estrategias que permitan optimizar los } \\
\text { procesos de toma de decisión en los ámbitos local y regional. } \\
\text { La cooperación, el trabajo en equipo y la coordinación de } \\
\text { grupos de investigación. } \\
\text { Conducir proyectos de investigación aplicada en el campos de } \\
\text { los estudios regionales. }\end{array}$ & $\begin{array}{l}\text { Desarrollo de trabajs originales, delimitación, } \\
\text { identificación y evaluación de la literatura, } \\
\text { aplicación de metodologías y generación } \\
\text { de conclusiones. } \\
\text { Aplicación de métodos, técnicas y } \\
\text { procedimientos de investigación. }\end{array}$ \\
\hline Proponer esquemas de evaluación de plitícas territoriales. & $\begin{array}{l}\text { Deberán presentar como característica la } \\
\text { multidisciplina para ser aplicadas al análisis } \\
\text { de lo municipal. }\end{array}$ \\
\hline
\end{tabular}

Fuente: elaboración propia con base en información del área de docencia MDR-MCs.

\section{territarios 34}

nicas y procedimientos de investigación; (b) desarrollo de trabajos originales, delimitación, identificación y evaluación de literatura, aplicación de metodologías y generación de conclusiones; (c) estas habilidades deberán mostrar como característica la multidisciplina para ser aplicadas al análisis de lo municipal. Por último, se hace referencia al campo de acción laboral donde el egresado como profesional puede aplicar sus conocimientos. 
La entrevista a profundidad se hizo a un egresado de la $7 .^{a}$ promoción y su análisis de experiencia se hizo a partir de los ejes temáticos señalados con anterioridad.

Visión consolidada: Para el egresado del Caso B la visión consolidada sobre la importancia de las ciencias sociales en la transformación de la vida colectiva no la adquirió en la MCS sino en otra que había realizado anteriormente (la Maestría en Urbanismo en Investigación y Docencia de la UNAM). Señala que el conocimiento para él

[...] no es un paquete que se te entrega cuando pasas por unos salones de clase, se logra como un proceso, y se logra en la interacción del tema con el trabajo, hay un devenir del aula a la actividad profesional y viceversa y en este devenir se alcanza la consolidación de una visión [...] (Comunicación personal Caso B, 2014).

Las bases teóricas para esta visión consolidada no se crearon porque el programa así lo establece, sino que es parte de tu bagaje intelectual tanto previo como a posteriori a la maestría (Comunicación personal Caso $\mathrm{B}, 2014)$. Comenta que el tema de visión consolidada es un tema ambiguo que habría que reflexionarlo desde diferentes ámbitos.

En términos de conocimientos, el egresado manifiesta que lo más importante que tuvo de la maestría fueron las "herramientas técnicas" para la actividad profesional, bases metodológicas y técnicas que le ayudaron a su perfil profesional. Otros conocimientos importantes fueron su acercamiento a los estudios trasversales, tales como estudios de género, pobreza, medio ambiente, tercer sector, etc. que permearon su formación más general.

Enfoque interdisciplinario: El egresado menciona que los estudios en ciencias sociales permiten percatarse de que las áreas de estudio se permean y tienen que fragmentarse para poder abarcar la complejidad misma de la sociedad, después lo puedes poner en la práctica (Comunicación personal Caso B, 2014). Él practica en su trabajo la interdisciplina todo el tiempo, pues trata con antropólogos urbanos, sociólogos, economistas y, aunque el diálogo no es sencillo, busca referentes comunes. Las habilidades que se forman con el tiempo para ponerla en práctica tienen que ver con la reflexión, capacidad de discusión, deliberación, cogestión, y gobernanza; a nivel colectivo se habla de empatía social, reconocimiento del otro, y capacidad para escuchar.

Articulación de los egresados a las necesidades territoriales: Actualmente el egresado tiene un cargo como funcionario público a nivel estatal como director de desarrollo urbano. Su trabajo consiste en darle atención a los 113 municipios de Michoacán así como conocer su problemáticas e intentar darles solución, es parte de su quehacer cotidiano como profesional. El egresado comenta que si hubiera trabajado inmediatamente después de la maestría, podría estar más cercano al entendimiento de la problemática municipal, pues tendría más frescos los conocimientos especializados en la formación como finanzas municipales, o historia de los municipios en México. Sin territarios 34 231 
embargo, el doctorado al mismo tiempo que lo alejó de temáticas municipales le brindó oportunidad de formar su capacidad intelectual para comprender la complejidad de cómo se estructura la administración pública en México, y por qué el municipio presenta rezagos.

En el Caso B sus egresados, según se puede observar en la figura 8 , un $37 \%$ de las personas entrevistadas trabaja en Instituciones de Educación Superior impartiendo clases o realizando investigaciones; un 29\% continúa con el grado Superior y un $22 \%$ trabaja en gobiernos en puesto clave del territorio. En este sentido, los objetivos puestos por la maestría se han cumplido y las capacidades adquiridas se han expandido para la inserción tanto en el mundo laboral como en su incidencia en el territorio (en el entendido de que el $22 \%$, por ejemplo, que trabaja en gobierno está involucrado en el diseño, gestión, evaluación de políticas públicas) información que tal vez habría que corroborar más con futuras investigaciones.

\section{Reflexiones finales}

De lo expuesto emergen algunos puntos para resaltar y reflexionar, si bien, aún de manera muy incipiente, pero invitan a replantear algunas cuestiones.

Los dos casos estudiados evidencian ser estudios de posgrado de excelencia, los cuales han tratado, desde sus orígenes, responder a enfoques concretos, con una fuerte connotación vinculada al entorno. En el caso A, se trata de una maestría con amplio enfoque para el desarrollo transfronterizo y en el caso B se trata de una maestría cuya diferenciación radica precisamente en el objeto de especialización municipal.

La fundamentación de las actualizaciones de sus objetivos y programas siempre ha respondido a la disponibilidad para ir adaptando el diseño de ambas maestrías en función de los grandes cambios producidos en estos últimos años en la misma concepción del desarrollo y sus teorías. Sin embargo, debe destacarse que esta disponi-

Figura 8. Cargos Laborales de los egresados 2013, Caso B

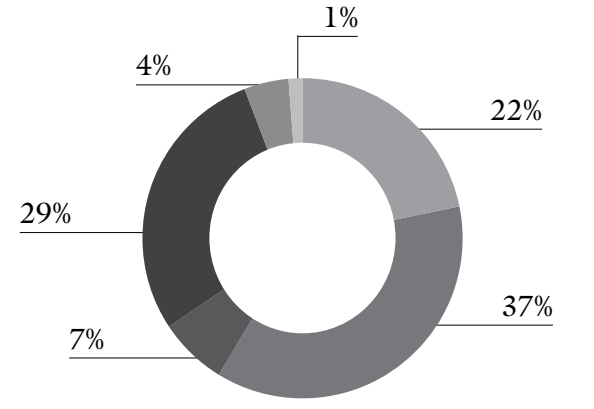

- Gobierno (19 egresados)

- Institución de Educación Superior (32 egresados)

- Organizaciones ( 6 egresados)

- Contaminación al grado superior (25 egresados)

- No laboran - sin información (4 egresados)

- Fallecidos (1 egresado)

Fuente: elaboración propia con base en información del área de docencia MCs. 
bilidad es producto de la discusión del núcleo académico que las conforma, quienes permean de manera inconsciente los ejes temáticos con sus tendencias y perspectivas. En este aspecto, se recomienda una mayor coordinación entre posgrados, de manera que pueda existir una mayor coherencia y complementariedad en la oferta educativa nacional.

La originalidad conceptual, por una parte, se expresa en superar el reduccionismo que ha sufrido tanto la conceptualización del territorio, concebido solo desde lo nacional, así como la del desarrollo anclada a una visión netamente economicista. Por otra parte, se debe acercar estos centros de estudio y sus egresados al territorio como un actor clave para acompañar procesos de desarrollo territorial. En este sentido, esto implica compartir con los demás actores del territorio oportunidades y fijar actuaciones prioritarias. Así mismo, orientar y formar especialistas, disponer de sistemas de evaluación de las políticas, contar con laboratorios de base de datos que nutran los distintos programas, entre muchas de las posibles actividades que se identificarán en el diseño de un proyecto de estudios de posgrados orientado a fomentar el vínculo universidad-territorio, que se manifiesta en la inserción de sus egresados en lugares clave para esta relación.

En cuanto al desarrollo de capacidades individuales, se considera que ambos programas por sus objetivos y metas, así como con la currícula y el lugar en el que los egresados desempeñan sus funciones dan cuenta de una inserción favorable, e indu- cen a pensar en la agencia de los egresados para intervenir y establecer su condición de actor insertado en el medio, funciones que denotan, entre otras, la generación de capacidades colectivas. Sin embargo, si se detiene la mirada en el análisis de los procesos de escalonamiento de capacidades, el pasaje de las capacidades individuales a las colectivas no es mecánico ni arbitrario, ya que siempre es necesario identificar los recursos que sostienen el andamiaje de dichas capacidades. Es tan cierto, que la capacitación recibida en estudios de posgrados es un recurso importante, tanto como la importancia atribuida a otros recursos que hacen posible que esta capacidad individual pueda expresarse (instituciones, poder económico, social, jurídico, político, etc.). Por otra parte, encarar el análisis de las capacidades colectivas y su influencia para generar desarrollo territorial implica pensar en la trayectoria de los propios actores (su formación anterior, por ejemplo), así como el modo en que las capacidades individuales repercuten sobre procesos efectivos (históricos) de gobierno y gestión de políticas públicas, aspectos múltiples y diversificados que no se abarcaron en este trabajo.

Lo anterior deja como reflexión que algunos de los egresados de maestrías con una visión local-territorial se enfrentan a mercados laborales que presentan estructuras corporativistas-centralistas. Su labor estratégica consiste en luchar por manifestar los problemas locales y hacerlos visibles en la agenda política de sus instituciones laborales. Les ha servido como herramienta de diálogo e integración, y su formación territarias 34 
integral-consolidada les ha permitido contar con instrumentos cognitivos que les permiten desarrollar proyectos profesionales con parámetros de calidad.

La inserción laboral de los egresados es buena y lo suficientemente relacionada con los objetivos de las maestrías, así como bastante diversificada. Sin embargo, se necesitan estudios más a largo plazo para poder evidenciar la trayectoria laboral, la permanencia en sus trabajos, así como la repercusión concreta que tienen sus acciones en el desarrollo territorial.

\section{Referencias}

Acuña, C. (Comp.) (2013). ¿Cuánto importan las instituciones? Gobierno, Estado y actores en la politica argentina ( $1 .^{\mathrm{a}} \mathrm{ed}$.). Buenos Aires: Siglo Veintiuno Editores.

Albuquerque, F., \& Dini, M. (2008). Guia de Aprendizaje sobre integración productiva y desarrollo económico territorial. Washington: Banco Interamericano de Desarrollo.

Almaraz, A. (2010). Actualización 20092010 Maestría en Desarrollo Regional $(M D R)$, Documento de Trabajo, Dirección General de Docencia, Institución Caso A.

Basabe, L., \& Cols, E. (2007). La enseñanza. De Camilloni, A. R., Cols, E., Basabe, L., \& Feeney, S., El saber didáctico (pp. 125162). Buenos Aires: Editorial Paidós.

Bebbington, A. (1999). Capitals and capabilities: a framework for analyzing tersitorias 34 234 poverty. World development, 27(12), 2021-2044.

Carreño, C. (2011). Posgrados sobre desarrollo en América Latina: origen y evolución. Educación y Educadores, 14(2), 327-345.

Costamagna, P. (2015). Innovación y Territorio. Nuevas actuaciones de las Agencias de Desarrollo. Prácticas y Estrategias de Cooperación Internacional para el DEL. FAMSCI Andalucía, España.

Costamagna, P., \& Pérez, R. (2013). Enfoque pedagógico para el Desarrollo Territorial y su relación con los espacios de formación (formales y no formales). Programa ConectaDEL. Recuperado de: http://www. conectadel.org/biblioteca- $2 /$ ?did $=264$ Costamagna, P., Pérez, R., \& Spinelli, E. (2013a). Elementos de un Enfoque Pedagógico para el Desarrollo Territorial. Programa ConectaDEL. Recuperado de: http://www.conectadel.org/biblioteca- $2 /$ ?did=265

Costamagna, P., Pérez, R., \& Spinelli, E. (2013b). Facilitadores de Procesos de Desarrollo Territorial: sistematización de antecedentes. Documento de discusión. Programa Conectadel. Recuperado de: http://www.conectadel.org/biblioteca-- $-2 /$ ?did $=263$

Díaz Alva, B. (2008). Formación ética y actuación pedagógica en la metodología de la enseñanza superior. V Encuentro Internacional de Kipus, Red de Docentes de América Latina y el Caribe. Recuperado de: http://www.redkipusperu. org/files/4.pdf 
García de Fanelli, A., Kent, S., Álvarez, G., \& Trombetta, A. (2001). Entre la academia y el Mercado: posgrados en ciencias sociales y politicas públicas en Argentina y México. México: Colección Biblioteca de la Educación superior, ANUIES.

Gibbons, M. (1998). Pertinencia de la educación superior en el siglo XXI. Washington: Banco Mundial.

Girardo, C. (2007). El desarrollo local en México: aportes teóricos y empiricos para el debate. México: UNAM- UADY.

Girardo, C. (2009). La construcción, apropiación y difusión del conocimiento en la profesionalización de agentes para el desarrollo local. En J. Basave \& M. Á. Rivera (Coords.), Globalización, conocimiento y desarrollo (pp. 101- 124). Tomo II, México: UnAM.

Girardo, C. (2012). La Educación y la Pedagogía para el desarrollo desde las organizaciones de la sociedad civil. Serie cuadernos de Investigación. Toluca-Estado de México: El Colegio Mexiquense.

Girardo, C., \& Suarez, M. H. (2010). El desarrollo regional y local: estado del arte en investigaciones financiadas y ofertas de formación en estudios superiores en México. En P. Vergara, SEBDERE Centros de Pensamiento Estratégico Territorial: instrumentos de la gobernanza regional en Chile (pp. 87-108). Chile: Ed. SEBDERE.

Greenwood, D. J., \& Levin, M. (2007). Introduction to Action Research - $2^{\text {nd }}$ Edition. Thousand Oaks: Sage Publications.
Kvale, S. (1983). The qualitative research interview: a phenomenological and hermeneutical mode of understanding. Journal Phenomenological psychology, 14, 171-196.

Madoery, O. (2014). Una reflexión politica sobre desarrollo y territorio. Centro de Estudios Desarrollo y Territorio. Recuperado de: http://www.cedet. edu.ar/Archivos/cuadernos/Cuaderno\%202\%20Reflexi\%C3\%B3n\%20 pol\%C3\%ADtica\%20sobre\%20desarro$110 \% 20 \mathrm{y} \% 20$ territorio.pdf

Martínez, S. (2005). Reestructuración de la Maestría en Desarrollo Regional 2005, Documento de trabajo, El Colegio de México.

Pereira, G. (2006). Capacidades individuales y capacidades colectivas. Sistema, 195, 35-51.

Perry, C. (1998). Processes of a case study methodology for postgraduate research in marketing. European Journal of Marketing, 32(9-10), 785-802.

Sanahuja, J. A. (2010). La construcción de una región. Suramérica y el regionalismo postliberal. En M. Cienfuegos \& J. A. Sanahuja (Eds.), La construcción de una región UNASUR y la integración en América del Sur (pp.87-134). Barcelona CIDOB: Balleterra.

Sen, A. (1993). The Quality of Life. Oxford: Clarendon.

Sen, A. (2002). Rationality and freedom. Cambridge, MA: Harvard University.

Singh, P. (2008). Oral and Written Reflection in Practice Management: An Action 


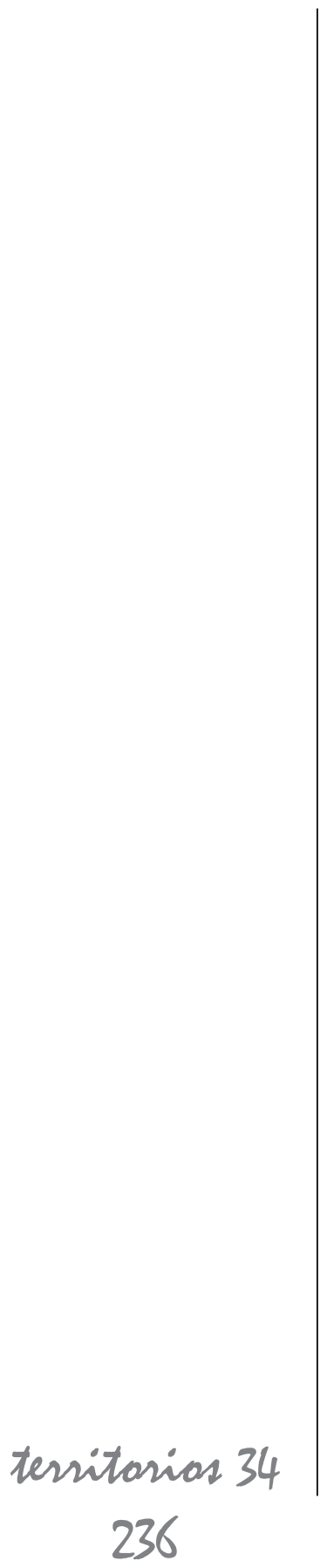

Research Approach. Systemic Practice and Action Research, 21(2), 171-185.

Steward, F. (2005). Groups and Capabilities. Journal of Human Development, 6(2), 185-204.
Storper, M. (1997). The regional world: territorial development in a global economy. New York: The Guilford Press.

Vázquez B. A. (2005). Las nuevas fuerzas del desarrollo. Barcelona: Antoni Bosch. 